\title{
Hydrodynamic Parameters in Various Methods of Soil Electrokinetic Treatment
}

\author{
Maxim Gucal $^{1}$, Veniamin Khyamyalyaynen ${ }^{1}$ \\ ${ }^{1}$ T.F. Gorbachev Kuzbass State Technical University, 650000, 28 Vesennyaya St., Kemerovo, Russia
}

\begin{abstract}
The features of determining the main hydrodynamic parameters of the process of soil electrokinetic treatment are considered. The main problem in determining these parameters is the complexity of the research and the necessity to use special laboratory equipment. One of the ways to solve this problem is proposed to use computer simulation of physical processes occurring in clay soils subjected to electroosmotic and electrochemical (ECP) processing. The results of calculations of the hydrodynamic parameters of the process of soils electrochemical treatment carried out according to the developed methodology of experimentalanalytical assessment of the clay soils electrical and hydrodynamic parameters according to the results of physical and computer modeling of processes in the area of electroosmotic processing are presented. The results of comparing hydrodynamic parameters calculated by computer simulation of unsteady physical processes occurring in clay soils during their electroosmotic and electrochemical processing are presented.
\end{abstract}

\section{Introduction}

The parameters characterizing the process of electroosmotic soil treatment are the speed of electroosmosis, the filtration coefficient, coefficients of electroosmosis and electroosmotic activity. Determination of these characteristics by various methods requires multistage cumbersome measurements, the availability of special laboratory equipment [1-8], therefore, it is advisable to increase the efficiency and accuracy by using the experimentalanalytical research method, including modeling of various stages of the experiment.

To obtain more detailed data, researchers often resort to mathematical modeling of various stages of a physical experiment. In particular, it is advisable to use computer modeling as a tool for such calculations as one of the most effective methods for studying of complex non-stationary systems. Investigating computer models is easier and more convenient than conducting real experiments, since the latter can be very labor-intensive and resource-intensive or their results can be unpredictable. Computer modeling helps to identify the main determinants of the properties of the studied object or process factors, allows you to predict the impact of the results from decisions made and to see the problem in its development.

Preliminary modeling of field experiments, which require significant expenditures, is one of the important directions in studies of unsteady physical processes occurring in soils. The result of such a preliminary assessment may be the substantiation of the parameters and 
operating modes of the developed experimental setup or their optimization, forecast and assessment of the expected results of the experiment.

The presented work is the result of modeling various processes of electrokinetic processing of soils and is aimed at determining the hydrodynamic parameters of processes occurring in soils.

\section{Results and discussion}

An algorithm for determining the basic hydrodynamic parameters of unsteady physical processes occurring in the zone of soil electrokinetic treatment [10-13] based on the experimental computer simulation technique is presented in [9].

The main informing parameter in this case is the specific electrical resistivity (SER) of soils, determined both by integral measurement schemes (georadar, electrical profiling and sounding), and by differentiated ones, which make it possible to obtain true SER (using microsensors, downhole induction and electrical logging).

In the analysis of geophysical data obtained using various schemes of geophysical data, the main difficulty remains the non-stationary nature of the processes occurring in soils, since the electro- and hydrodynamic parameters of the array continuously change during processing. To solve this problem, it is advisable to perceive a time-unsteady process as a sequential change in a number of stationary processes occurring at certain intervals, and evaluate the changes in the properties of the array according to the data of discrete measurements. This approach will increase the information content of monitoring, as well as evaluate changes in the properties of the array.

The main initial data, intermediate and final results of modeling the process of electroosmotic treatment of clay soil are given in [9].

At the initial stage of model creation, the assumptions given below are made:

- the relative dielectric constant of the original array has a value that corresponds to that adopted for wet clay;

- in the process of electrical treatment, three characteristic zones are formed with values of relative dielectric constant corresponding to clay in a wet, moistened and dry state;

- soil treatment modes, as well as the dimensions of the computer model, corresponded to the physical model.

The assumptions made subsequently contributed to the determination of the zones formed during the electrochemical treatment.

According to the technique adopted in [9], the main calculation parameter is the relative filtration velocity, which is equal to the modulus of the electric field

$$
V(x ; z) /_{k_{e}}=E(x ; z),
$$

where $V$ - the effective velocity of electroosmotic filtration, $\mathrm{m} / \mathrm{s} ; k_{e}$ - the coefficient of electroosmosis, $\mathrm{m}^{2} / \mathrm{V} \cdot \mathrm{s} ; E$ - the electric field strength, $\mathrm{V} / \mathrm{m}$.

Figure 1 shows graphs of the SER $\rho(x, I \cdot t)$, which represent the initial database of experimental data.

According to Figure 1, the velocity of change of the electric treatment process was determined. According to the methodology [9], the difference in the areas located under the graphs of relative SER is taken as the main characteristic of the change in the processes. 


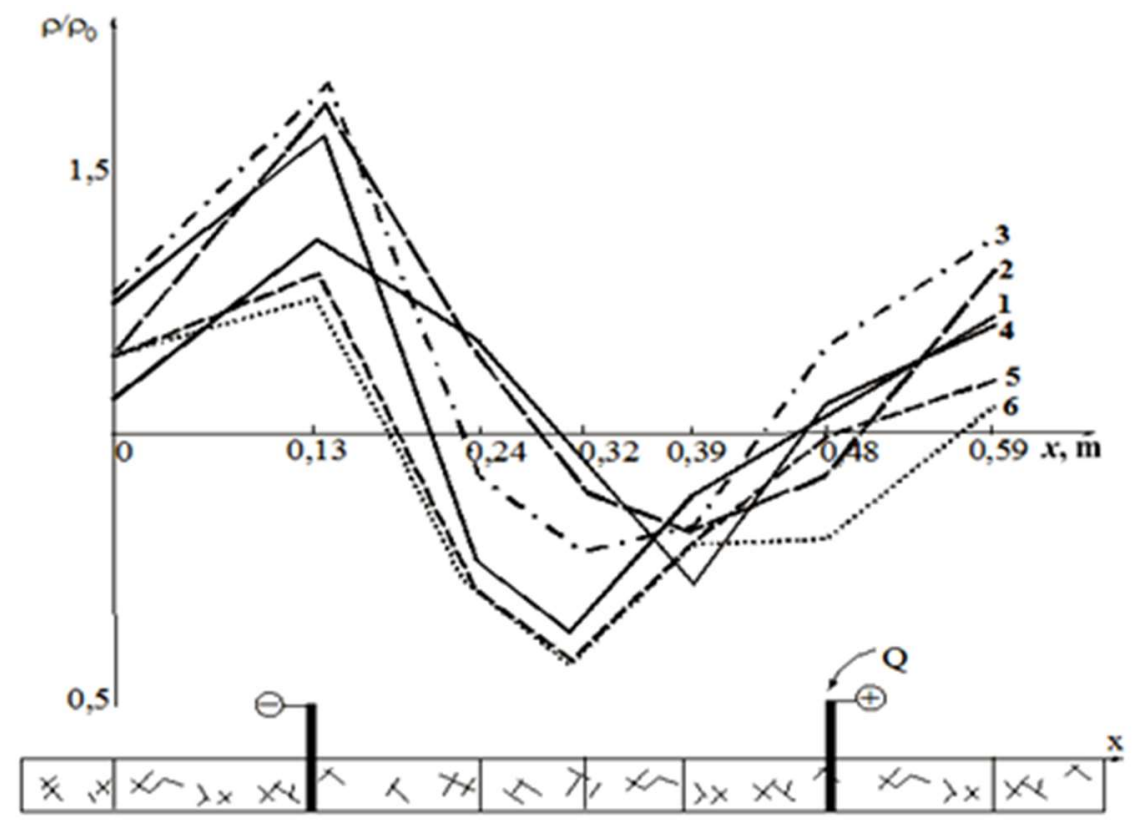

Fig. 1. Change in SER in the zone of electrochemical treatment: $1-I \cdot t=6$ A.h; $2-9 ; 3-12 ; 4-18$; $5-25 ; 6-30$

The velocity of change of the process is determined by the following relationship:

$$
V_{s i}=k \frac{\left(s_{i+1}-S_{i}\right)}{s_{i}}
$$

where $S_{i+1}$ and $S_{i}$ - corresponding to the subsequent and previous time of measuring the area under the graphs of the relative SER $\rho / \rho_{0} ; k$ - the correction factor determined from the ratio of the reference velocity and calculated by the formula (2) (Figure 2) [9].

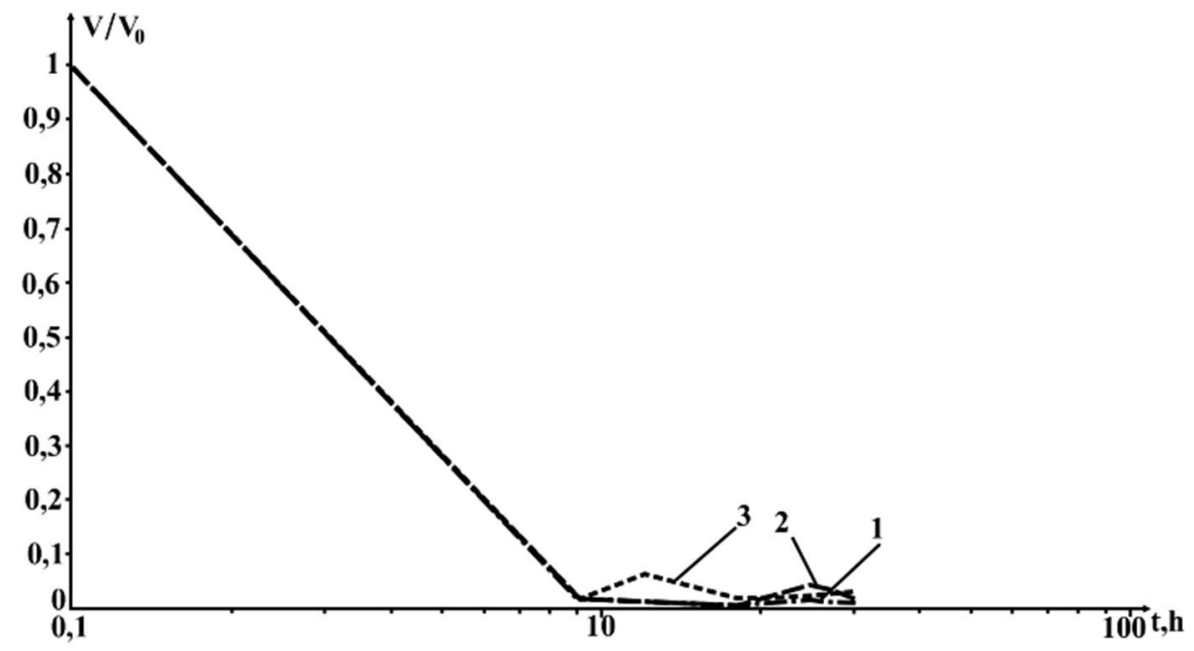

Fig. 2. Relative velocity of electrochemical fixing:1, 2, 3 - calculated velocity in, respectively, the zone of saturation with a chemical solution, the intermediate zone and the zone of moisture accumulation 
Figure 3 shows the graphs of the intensity of the process of electrochemical fixing, obtained during the simulation of the experimental installation [9] when using a set of programs that implement the finite element method.

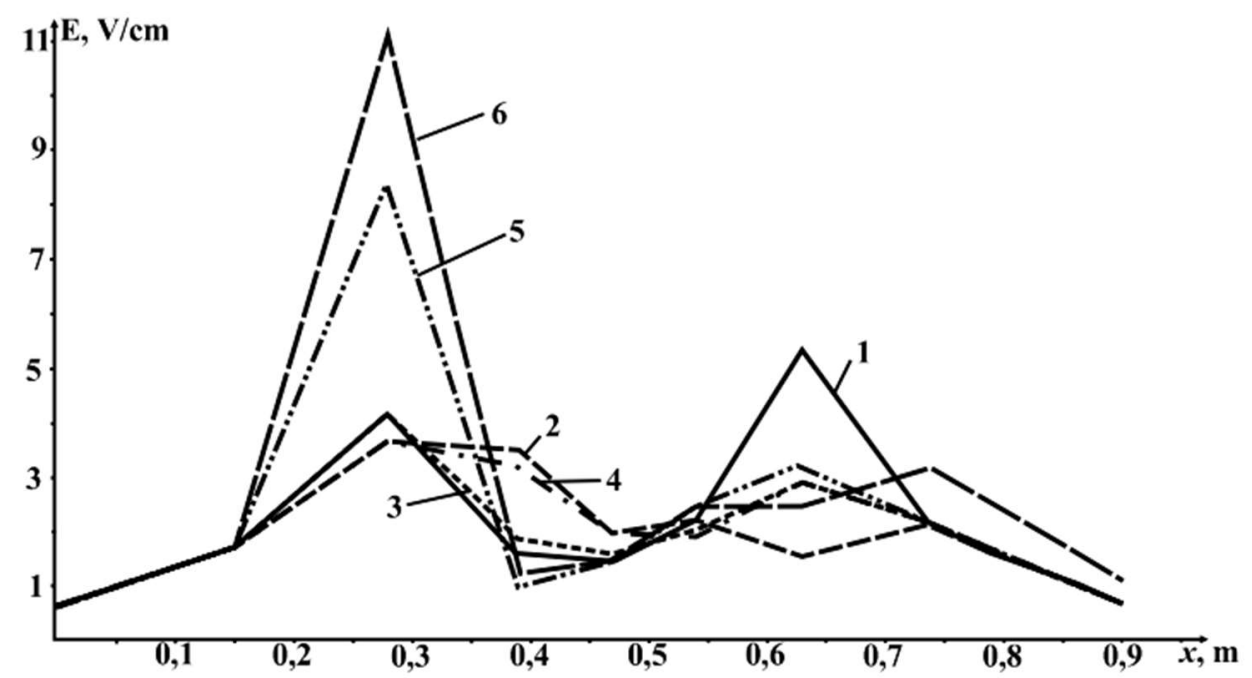

Fig. 3. Electric field intensity during electrochemical fixing as a function of time at depth $z=0,125$ $\mathrm{m}:$

$1-\mathrm{I} \cdot \mathrm{t}=6 \mathrm{~A} \cdot \mathrm{h} ; 2-9 ; 3-12 ; 4-18 ; 5-25 ; 6-30$

The filtration coefficient was determined using the dependence [9]

$$
V=k_{f} \cdot \operatorname{grad} P=k_{f} \cdot \frac{\Delta P}{\Delta x},
$$

where $V$ - the velocity, $\mathrm{cm} / \mathrm{s} ; k_{f}$ - the filtration coefficient, $\mathrm{cm} / \mathrm{s} ; \Delta P$ - the pressure drop, $\mathrm{cm}$; $\Delta x$ - the length of the filtration path, $\mathrm{cm}$.

The pressure drop $\Delta P$ and the filtration path length $\Delta x$ were determined using the simulation results (Figure 3) [9], from which the equality $\left|\Delta \mathrm{P}_{\mathrm{i}}\right| \sim\left|\Delta \varphi_{\mathrm{i}}\right|$.

Figure 4 shows graphs of changes in the filtration coefficient of the process of electrochemical fixing.

From the graphs shown in Figures 2 and 4, it can be said that the process of electrochemical treatment of the soil, as well as the processes occurring in the zone of electroosmotic processing [9], are characterized by a decrease in the calculated values of velocity and filtration coefficient over time. 


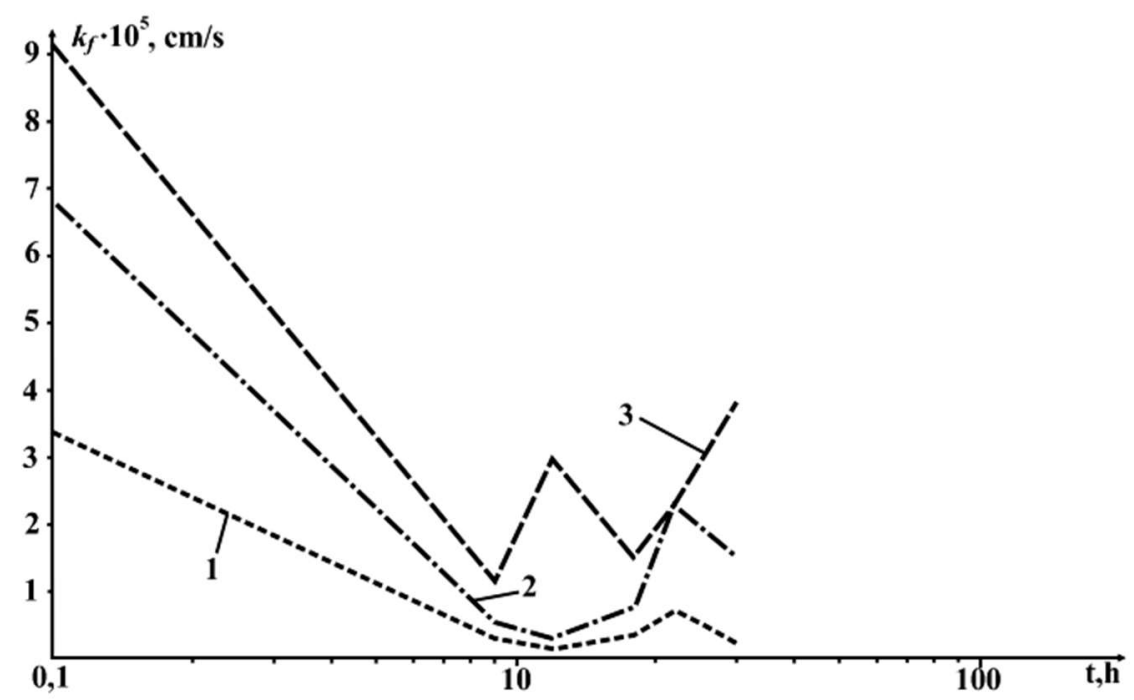

Fig. 4. The dependence of the filtration coefficient $k_{f}$ on time during electrochemical fixing: 1 calculation in the drainage zone (saturation with a chemical solution); 2 - calculation in the transition zone; 3 - calculation in the zone of moisture accumulation

\section{Conclusion}

From the above graphs it follows that the electroosmotic process proceeds more intensively than the electrochemical one. This can be explained by the fact that the filtration velocity of a concentrated and, as a result, more viscous chemical solution compared to natural groundwater in relatively moist clay soil is relatively low. This can be seen from a comparison of the dependences shown in Figs. 2 and 4 with the calculation results given in [9], where it is shown that with electrochemical fixing, the rate of the process (as the natural moisture is replaced by a chemical solution) decreases faster than with electroosmosis.

Also, differences in the dynamics of processes are visible from a comparison of changes in the filtration coefficient. The character of the dependences of the coefficient $\mathrm{kf}$ with the course of the processes of electroosmosis and ECP is preserved. The differences in the filtration coefficient can be explained by the fact that during ECP not only the process of electroosmotic drainage occurs, but also the soil is moistened with a chemical solution due to the replacement of a natural liquid. Although, most likely, after removing the load on the electrodes, in the process of gaining an array of strength, the filtration coefficient will continue to decline.

To establish the optimal course of the controlled ECP process, it is necessary to carefully select the concentration of the working chemical solution.

\section{References}

1. D. V. Morris, S. F. Hillis, J. A. Caldwell, Can. Geotech. J., 22, 8 (1985)

2. S. Hansbo, Sixth International Conference on Case Histories in Geotechnical Engineering, 15 (2008)

3. R.Z. Velten, D.C. Lima, M.P.F. Fontes, C.A.B. Carvalho, Soils and Rocks, 32, 11 (2012) 
4. Shao-Chi Chiena, Pio-Go Hsiehb, Chang-Yu Ou, ACEM 12, 11 (2012)

5. Y. Guo, Electrokinetic dewatering of oil sands tailings, 3, 112 (2012)

6. V. dos Santos, M. O. Medeiros, A. S. D. dos Anjos, C. A. Martínez-Huitle, D. R. da Silva, Chemical Engineering Transactions, 41, 6 (2014)

7. S. M. Prostov, E. A. Shabanov, The 8 th Russian-Chinese Symposium. Coal in the $21 \mathrm{st}$ Century: Mining, Processing and Safety, 1, 9 (2016)

8. F. Eriksson, L. Gemvik, Electro-Osmotic Treatment of Soil, 1, 116 (2014)

9. M. Gucal, A. Pokatilov, E3S Web Conf., 105, 01012 (2019)

10. J. Yuan, M.A. Hicks, J. Dijkstra, ISSMGE - TC 211, 8 (2012)

11. S. R. Maduar, A. V. Belyaev, V. Lobaskin, O. I. Vinogradova, PRL, 114, 5 (2015)

12. Zhi-ming Liu, Jian-gui Yang, Qi-meng Li, EJGE, 21, 13 (2016) 